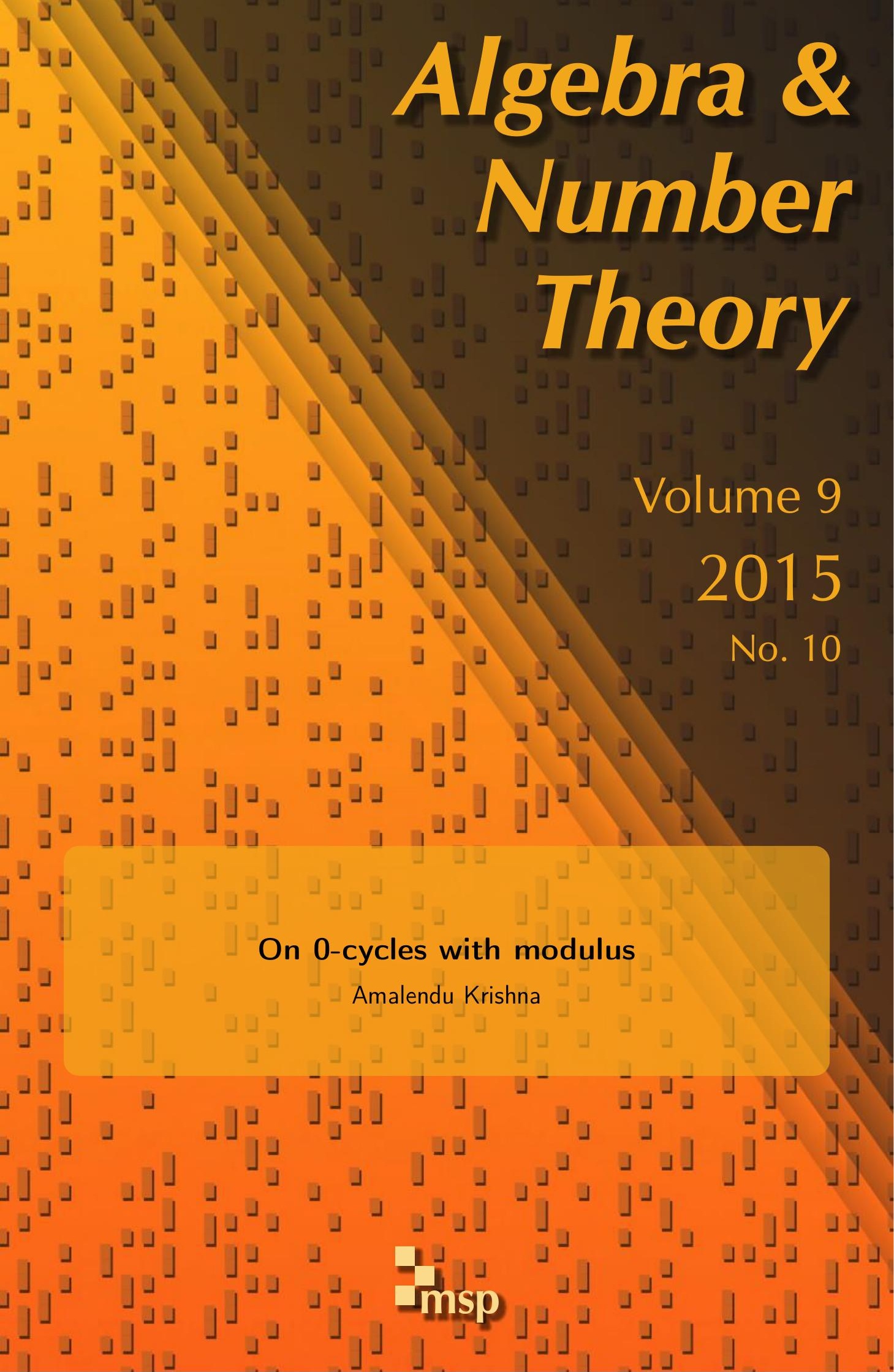




\title{
On 0-cycles with modulus
}

\author{
Amalendu Krishna
}

\begin{abstract}
Given a nonsingular surface $X$ over a field and an effective Cartier divisor $D$, we provide an exact sequence connecting $\mathrm{CH}_{0}(X, D)$ and the relative $K$-group $K_{0}(X, D)$. We use this exact sequence to answer a question of Kerz and Saito whenever $X$ is a resolution of singularities of a normal surface. This exact sequence and two vanishing theorems are used to show that the localization sequence for ordinary Chow groups does not extend to Chow groups with modulus. This in turn shows that the additive Chow groups of 0 -cycles on smooth projective schemes cannot always be represented as reciprocity functors.
\end{abstract}

\section{Introduction}

The idea of algebraic cycles with modulus was first conceived by Bloch and Esnault [2003b; 2003a]. One main motivation behind such a theory is to develop a theory of motivic cohomology which can describe the relative $K$-theory of smooth schemes relative to closed subschemes. A potential candidate for such a theory was later constructed and studied by Park [2009], Krishna and Levine [2008] and more recently by Kerz and Saito [2015] and Binda and Saito [2014]. It was conjectured in [Krishna and Levine 2008] that there should exist a spectral sequence consisting of these motivic cohomology groups whose abutment is the relative $K$-theory.

The results of this text were partly motivated by the following question of Kerz and Saito [2015, Question V]. Let $X$ be a smooth quasiprojective scheme of dimension $d$ over a field $k$ and let $D \hookrightarrow X$ be an effective Cartier divisor. Let $\mathrm{CH}_{0}(X, D)$ denote the Chow group 0 -cycles on $X$ with modulus $D$. Let $\mathcal{K}_{d,(X, D)}^{M}$ denote the relative Milnor $K$-theory sheaf on $X$. Let $U$ be an open subscheme of $X$ whose complement is a divisor.

Question 1.1. Assume that $X$ is projective and $k$ is a perfect field of positive characteristic. Is there an isomorphism

$$
\underset{D}{\lim } \mathrm{CH}_{0}(X, D) \stackrel{\sim}{\longrightarrow} \underset{D}{\lim } H_{\text {nis }}^{d}\left(X, \mathcal{K}_{d,(X, D)}^{M}\right),
$$

where the limits are taken over all effective divisors on $X$ with support outside $U$ ?

MSC2010: primary 14C25; secondary 14F30, 14G40.

Keywords: algebraic cycles, modulus condition, $K$-theory. 
It follows from the main results of [Kato and Saito 1986; 2015] and [Rülling and Saito 2015] that this question has a positive solution if $k$ is finite and the support of $X \backslash U$ is a normal crossing divisor. As explained in [Kerz and Saito 2015], the above question is part of the bigger question of whether the Chow groups with modulus satisfy Nisnevich or Zariski descent. As we shall see shortly, the above question is also directly related to the conjectured connection between the cycles with modulus and the relative $K$-theory.

Main results. Let $\operatorname{Pic}(X, D)$ denote the isomorphism classes of pairs $(\mathcal{L}, \phi)$, where $\mathcal{L}$ is a line bundle on $X$ and $\phi$ is an isomorphism $\phi:\left.\mathcal{L}\right|_{D} \stackrel{\sim}{\longrightarrow} \mathcal{O}_{D}$. We prove the following result as a partial answer to the above question.

Theorem 1.2. Let $k$ be any field and let $X$ be a nonsingular quasiprojective surface over $k$ with an effective Cartier divisor D. Then there is an exact sequence

$$
\mathrm{CH}_{0}(X, D) \stackrel{\operatorname{cyc}_{(X, D)}}{\longrightarrow} K_{0}(X, D) \longrightarrow \operatorname{Pic}(X, D) \longrightarrow 0 .
$$

In particular, $\operatorname{cyc}_{(X, D)}$ induces a surjective map $\mathrm{CH}_{0}(X, D) \rightarrow H_{\mathrm{nis}}^{2}\left(X, \mathcal{K}_{2,(X, D)}^{M}\right)$.

Remark 1.3. The map $\operatorname{cyc}_{(X, D)}$ turns out to be injective as well if $X$ is affine. A proof of this using completely different type of argument will appear in [Binda and Krishna $\geq 2015$ ].

Let us now assume that $X$ is a resolution of singularities of a normal surface $Y$ and let $U$ denote the regular locus of $Y$. Then we can use Theorem 1.2 to obtain the following finer result which fully answers Question 1.1 for a special class of surfaces.

Theorem 1.4. Let $k$ be any field and let $X$ be a resolution of singularities of a normal surface $Y$. Let $U$ denote the regular locus of $Y$. Then the cycle class map $\mathrm{CH}_{0}(X, D) \rightarrow H_{\text {nis }}^{2}\left(X, \mathcal{K}_{2,(X, D)}^{M}\right)$ induces an isomorphism

$$
\lim _{D} \mathrm{CH}_{0}(X, D) \stackrel{\sim}{\longrightarrow} \underset{D}{\lim } H_{\text {nis }}^{2}\left(X, \mathcal{K}_{2,(X, D)}^{M}\right)
$$

where the limits are taken over all effective divisors on $X$ with support outside $U$.

Localization sequence for Chow groups with modulus. Since the introduction of the Chow groups with modulus, various authors have been trying to prove several properties of these Chow groups which are analogous to the well-known properties of Bloch's higher Chow groups. It was shown in [Krishna and Park 2014] recently that the Chow groups with modulus satisfy projective bundle and blowup formulas. It was however not known if the localization sequence for Bloch's higher Chow groups is true for Chow groups with modulus. We use Theorem 1.2 to show that the Chow groups with modulus do not admit such a localization sequence. In fact, we show that even the localization sequence for the ordinary Chow groups (in the 
sense of [Fulton 1998]) does not admit extension to Chow groups with modulus. Answering this question was another motivation of this note.

Let $m \geq 2$ be any integer and let $D$ denote the Cartier divisor $\operatorname{Spec}\left(k[t] /\left(t^{m}\right)\right)$ inside $\operatorname{Spec}(k[t])$. For any $Y \in \mathbf{S c h} / k$, the Cartier divisor $Y \times D \hookrightarrow Y \times \mathbb{A}_{k}^{1}$ is denoted by $D$ itself.

Theorem 1.5. Let $k$ be an algebraically closed field of characteristic zero with infinite transcendence degree over $\mathbb{Q}$. Let $Y$ be a connected projective curve over $k$ of positive genus. Then for any inclusion $i:\{P\} \hookrightarrow Y$ of a closed point, the sequence

$$
\mathrm{CH}_{0}\left(\{P\} \times \mathbb{A}_{k}^{1}, D\right) \stackrel{i_{*}}{\longrightarrow} \mathrm{CH}_{0}\left(Y \times \mathbb{A}_{k}^{1}, D\right) \stackrel{j^{*}}{\longrightarrow} \mathrm{CH}_{0}\left(Y \backslash\{P\} \times \mathbb{A}_{k}^{1}, D\right) \longrightarrow 0
$$

is not exact.

In particular, the localization sequence for Bloch's higher Chow groups does not extend to the Chow groups with modulus, even for a closed pair of smooth schemes.

The proof of this negative result is based on Theorem 1.2 and the following two vanishing theorems of independent interest.

Theorem 1.6. Let $k$ be any field and let $Y$ be any nonsingular affine scheme over $k$ of dimension $d \geq 1$. Then $\mathrm{CH}_{0}\left(Y \times \mathbb{A}_{k}^{1}, D\right)=0$.

Theorem 1.7. Let $k$ be an algebraic closure of a finite field and let $X$ be a smooth affine scheme over $k$ of dimension $d \geq 3$. Then for any effective Cartier divisor $D \hookrightarrow X$, we have $\mathrm{CH}_{0}(X, D)=0$. Assuming $D_{\text {red }}$ is a normal crossing divisor, we also have $H_{\text {nis }}^{d}\left(X, \mathcal{K}_{d,(X, D)}^{M}\right)=0$.

Remark 1.8. Theorem 1.7 implies that the analogue of Question 1.1 has a positive solution for affine schemes over $k$ of dimension at least three if $D_{\text {red }}$ is a normal crossing divisor.

Remark 1.9. The assertion of Theorem 1.7 is true also for $d=2$ and will appear in [Binda and Krishna $\geq 2015$ ]. The proof in this note does show at least that $\mathrm{CH}_{0}(X, D)_{\mathbb{Q}}=0$ even if $X$ is a surface.

On the other hand, it is easily seen using the surjection $\mathrm{CH}_{0}(X, D) \rightarrow \mathrm{CH}_{0}(X)$ that $d \geq 2$ is a necessary condition for the vanishing of $\mathrm{CH}_{0}(X, D)$.

Additive Chow groups and reciprocity functors. Ivorra and Rülling [ $\geq 2015]$ introduced the reciprocity functors $T\left(\mathcal{M}_{1}, \cdots, \mathcal{M}_{r}\right)$. These reciprocity functors are expected to describe the ordinary as well as the additive higher Chow groups of 0 -cycles for smooth projective schemes over a field. In this direction, it was shown by Ivorra and Rülling [ $\geq 2015$, Corollary 5.2.5] that for a smooth projective scheme $X$ of dimension $d$ over a field $k$, there is an isomorphism $T\left(\mathbb{G}_{m}^{\times r}, \mathrm{CH}_{0}(X)\right)(k) \simeq$ $\mathrm{CH}^{d+r}(X, r)$. They also show that $T\left(\mathbb{G}_{a}, \mathrm{CH}_{0}(\operatorname{Spec}(k))\right)(k) \simeq \mathrm{CH}_{0}\left(\mathbb{A}_{k}^{1}, D_{2}\right)$ if $\operatorname{char}(k)=0$, where $D_{2}=\operatorname{Spec}\left(k[t] /\left(t^{2}\right)\right)$. This was a verification of a special case of the general expectation that $T\left(\mathbb{G}_{a}, \mathrm{CH}_{0}(X)\right)(k)$ should be isomorphic to 
the additive Chow group $\mathrm{CH}_{0}\left(X \times \mathbb{A}_{k}^{1}, D_{2}\right)$ if $X$ is a smooth projective scheme over $k$. However, combining Theorems 1.5 and 1.6 with [Rülling and Yamazaki 2014, Theorem 1.1], we prove:

Corollary 1.10. Let $k$ be an algebraically closed field of characteristic zero with infinite transcendence degree over $\mathbb{Q}$. Let $Y$ be a connected projective curve over $k$ of positive genus. Then $\mathrm{CH}_{0}\left(Y \times \mathbb{A}_{k}^{1}, D_{2}\right)$ cannot be described in terms of the reciprocity functors.

Outline of proofs. We recall the definitions of Chow groups with modulus in Section 2. We then use the Thomason-Trobaugh spectral sequence to relate the cohomology of the sheaf $\mathcal{K}_{2,(X, D)}^{M}$ with the relative $K$-groups. We first prove an analogue of Theorem 1.2 for curves in Section 3 and deduce it for surfaces using Lemma 3.2. The proof of Theorem 1.2 is completed using some results of [Kato and Saito 1986] and Theorem 1.4 proven by using a combination of Theorem 1.2 and an explicit formula for the Chow group of 0 -cycles on normal surfaces from [Krishna and Srinivas 2002].

We prove Theorem 1.6 by first reducing to the case of curves. This case is achieved with the help of an algebraic version of a sort of containment lemma. We prove Theorem 1.5 as a combination of Theorems 1.2 and 1.6. This reduces the problem to understanding a map of cohomology groups of the relative $K$-theory sheaves of nilpotent ideals. This in turn can be written as an explicit map of $k$-vector spaces, where $k$ is the ground field. Theorem 1.7 is proven by reducing to the case of affine surfaces and empty Cartier divisor using some Bertini theorems.

\section{Recollection of Chow group with modulus and relative $K$-theory}

We fix a field $k$ and let $\mathbf{S c h} / k$ denote the category of quasiprojective schemes over $k$. Let $\mathbf{S m} / k$ denote the full subcategory of $\mathbf{S c h} / k$ consisting of nonsingular (regular) schemes. Given $X \in \mathbf{S c h} / k$, we shall write $X_{\text {sing }}$ and $X_{\text {reg }}$ for the closed and open subschemes of $X$, where $X_{\text {red }}$ is singular and regular, respectively. In this text, a curve will mean an equidimensional quasiprojective scheme over $k$ of dimension one. For a curve $C$, the scheme $C^{N}$ will often denote the normalization of $C_{\text {red }}$. Given a closed immersion $Y \hookrightarrow X$ in $\mathbf{S c h} / k$, we let $|Y|$ denote the support of $Y$ with the reduced induced closed subscheme structure.

For $X \in \mathbf{S c h} / k$, let $K(X)$ and $G(X)$ denote the $K$-theory spectra of perfect complexes and coherent sheaves on $X$, respectively. For a closed subscheme $Y \hookrightarrow X$, let $K(X, Y)$ denote the homotopy fiber of the restriction map $K(X) \rightarrow K(Y)$. For a sheaf $\mathcal{F}$ on the small Zariski (resp. Nisnevich) site of $X$, let $H_{\text {zar }}^{*}(X, \mathcal{F})$ (resp. $H_{\text {nis }}^{*}(X, \mathcal{F})$ ) denote the cohomology groups of $\mathcal{F}$. A cohomology group in this text without mention of the underlying site will indicate the Zariski cohomology. 
Thomason-Trobaugh spectral sequence for $K$-theory with support and relative $\boldsymbol{K}$-theory. Given a scheme $X$ and a closed subscheme $Y \hookrightarrow X$, let $K^{Y}(X)$ denote the homotopy fiber of the restriction map of spectra $K(X) \rightarrow K(X \backslash Y)$. Let $\mathcal{K}_{i,(X, Y)}$ denote the Zariski sheaf on $X$ whose stalk at a point $x \in X$ is the relative group $K_{i}\left(\mathcal{O}_{X, x}, \mathcal{O}_{Y, x}\right)$ for $i \in \mathbb{Z}$. Given a closed point $x \in X_{\text {reg }} \backslash Y$, the spectrum $K^{\{x\}}(Y)$ is contractible and hence there are natural maps of spectra

$$
K(k(x)) \rightarrow K^{\{x\}}(X) \rightarrow K(X, D) \rightarrow K(X) .
$$

In particular, there is a commutative diagram of Thomason-Trobaugh spectral sequences [1990, Corollary 10.5]

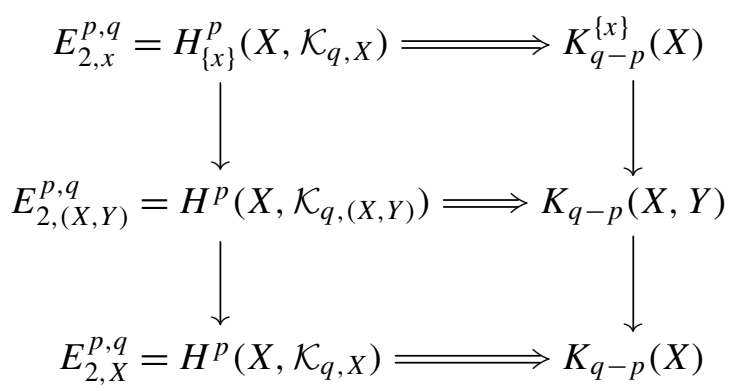

which is valid even when the Zariski cohomology is replaced by the Nisnevich cohomology.

Lemma 2.1. Given a modulus pair $(X, D)$ of dimension two over $k$, there is a short exact sequence

$$
0 \longrightarrow H_{\mathcal{C}}^{2}\left(X, \mathcal{K}_{2,(X, D)}\right) \longrightarrow K_{0}(X, D) \longrightarrow \operatorname{Pic}(X, D) \longrightarrow 0
$$

where $\mathcal{C}$ is either Zariski or Nisnevich cohomology. In particular, the map $H_{\mathrm{zar}}^{2}\left(X, \mathcal{K}_{2,(X, D)}\right) \rightarrow H_{\mathrm{nis}}^{2}\left(X, \mathcal{K}_{2,(X, D)}\right)$ is an isomorphism.

Proof. Let $\mathcal{C}$ denote either the Zariski or the Nisnevich cohomology. Since the $\mathcal{C}$-cohomological dimension of $X$ is two, the strongly convergent spectral sequence $E_{2}^{p, q}=H_{\mathcal{C}}^{p}\left(X, \mathcal{K}_{q,(X, D)}\right) \Rightarrow K_{q-p}(X, D)$ with differential $d_{r}: E_{r}^{p, q} \rightarrow E_{r}^{p+r, q+r-1}$ gives us an exact sequence

$$
H_{\mathcal{C}}^{0}\left(X, \mathcal{K}_{1,(X, D)}\right) \stackrel{d_{2}^{0,1}}{\longrightarrow} H_{\mathcal{C}}^{2}\left(X, \mathcal{K}_{2,(X, D)}\right) \longrightarrow K_{0}(X, D) \longrightarrow H_{\mathcal{C}}^{1}\left(X, \mathcal{K}_{1,(X, D)}\right) \longrightarrow 0 .
$$

By Hilbert's theorem 90, the map $H_{\text {zar }}^{1}\left(X, \mathcal{K}_{1,(X, D)}\right) \rightarrow H_{\text {nis }}^{1}\left(X, \mathcal{K}_{1,(X, D)}\right)$ is an isomorphism and it follows from [Suslin and Voevodsky 1996, Lemma 2.1] that $H_{\text {zar }}^{1}\left(X, \mathcal{K}_{1,(X, D)}\right) \stackrel{\sim}{\longrightarrow} \operatorname{Pic}(X, D)$. We are thus left with showing that $d_{2}^{0,1}=0$. We prove this for the Zariski cohomology as the same argument applies in the Nisnevich case. 
Applying the above spectral sequence for $K_{1}(X, D)$, the equality $d_{2}^{0,1}=0$ is equivalent to the assertion that the map $K_{1}(X, D) \rightarrow H^{0}\left(X, \mathcal{K}_{1,(X, D)}\right)$ is surjective. To prove this, we let $f \in H^{0}\left(X, \mathcal{K}_{1,(X, D)}\right)$. This is equivalent to a regular map $f: X \rightarrow \mathbb{G}_{m}$ such that $\left.f\right|_{D}=1$ and hence to a commutative diagram with exact rows

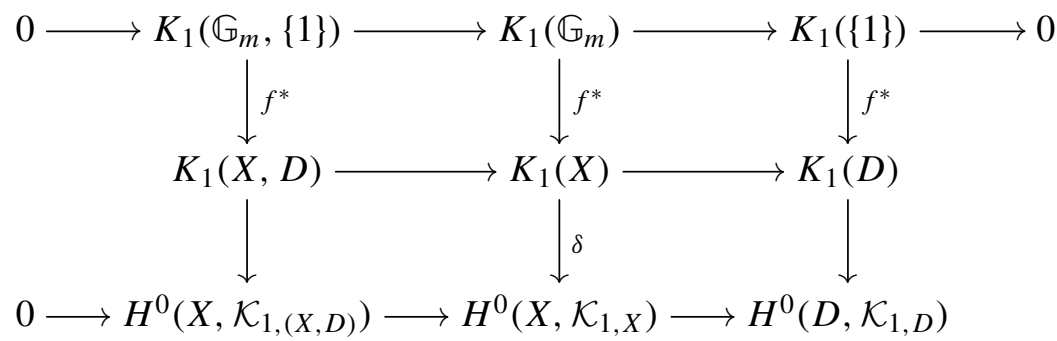

If we let $\mathbb{G}_{m}=\operatorname{Spec}\left(k\left[t^{ \pm 1}\right]\right)$, then one can check (as is well known) that $\delta \circ f^{*}([t])=f$. Since $t \in K_{1}\left(\mathbb{G}_{m},\{1\}\right)$, we see that $f^{*}([t]) \in K_{1}(X, D)$ and $\delta \circ f^{*}(t)$ dies in $H^{0}\left(D, \mathcal{K}_{1, D}\right)$. Hence, it must lie in $H^{0}\left(X, \mathcal{K}_{1,(X, D)}\right)$. It follows that the map $K_{1}(X, D) \rightarrow H^{0}\left(X, \mathcal{K}_{1,(X, D)}\right)$ is surjective.

Remark 2.2. The isomorphism $H_{\text {zar }}^{2}\left(X, \mathcal{K}_{2,(X, D)}\right) \stackrel{\sim}{\longrightarrow} H_{\text {nis }}^{2}\left(X, \mathcal{K}_{2,(X, D)}\right)$ was shown earlier by Kato and Saito [1986, Proposition 9.9] by a different method.

Chow groups of 0-cycles with modulus. We recall the definition of the Chow group of 0-cycles with modulus (see [Binda and Saito 2014, §2] or [Krishna and Park 2014, §2]).

Let $X$ be a nonsingular scheme of pure dimension $d$ and let $D \subsetneq X$ be an effective Cartier divisor on $X$. We shall call such a pair $(X, D)$ of a nonsingular scheme and an effective Cartier divisor, a $d$-dimensional modulus pair. Let $\mathcal{Z}_{0}(X, D)$ denote the free abelian group on the closed points in $X \backslash D$. Let $C \hookrightarrow X \times \mathbb{P}_{k}^{1}$ be a closed irreducible curve satisfying:

(1) $C$ is not contained in $X \times\{0,1, \infty\}$.

(2) If $v: C^{N} \rightarrow X \times \mathbb{P}_{k}^{1}$ denotes the composite map from the normalization of $C$, then one has an inequality of Weil divisors on $C^{N}$ :

$$
v^{*}\left(D \times \mathbb{P}_{k}^{1}\right) \leq v^{*}(X \times\{1\}) .
$$

We call such curves admissible. Let $\mathcal{Z}_{1}(X, D)$ denote the free abelian group on admissible curves and let $\mathcal{R}_{0}(X, D)$ denote the image of the boundary map $\left(\partial_{0}-\partial_{\infty}\right): \mathcal{Z}_{1}(X, D) \rightarrow \mathcal{Z}_{0}(X, D)$. The Chow group of 0 -cycles on $X$ with modulus $D$ is defined as the quotient

$$
\mathrm{CH}_{0}(X, D):=\frac{\mathcal{Z}_{0}(X, D)}{\mathcal{R}_{0}(X, D)}
$$


To relate this definition of $\mathrm{CH}_{0}(X, D)$ with the one given by Kerz and Saito [2015], let $\pi_{C}: C^{N} \rightarrow C$ denote the normalization of an integral curve $C \hookrightarrow X$ which is not a component of $D$. Let $A_{C \mid D}$ and $A_{C^{N} \mid D}$ denote the semilocal rings of $C$ and $C^{N}$ at the supports of $C \cap D$ and $\pi_{C}^{-1}(C \cap D)$, respectively. Let $\mathcal{R}_{0}^{\prime}(X, D)$ denote the subgroup of $\mathcal{Z}_{0}(X, D)$ given by the image

$$
\coprod_{C \not \subset D} K_{1}\left(A_{C^{N} \mid D}, I_{D}\right) \stackrel{\text { div }}{\longrightarrow} \mathcal{Z}_{0}(X, D) .
$$

Note that the surjectivity of the map $K_{2}\left(A_{C^{N} \mid D}\right) \rightarrow K_{2}\left(\pi_{C}^{*}(D)\right)$ implies that

$$
\begin{aligned}
K_{1}\left(A_{C^{N} \mid D}, I_{D}\right) & =\operatorname{Ker}\left(K_{1}\left(A_{C^{N} \mid D}\right) \rightarrow K_{1}\left(\pi_{C}^{*}(D)\right)\right. \\
& =\underset{U}{\lim } \operatorname{Ker}\left(\mathcal{O}(U)^{\times} \rightarrow \mathcal{O}\left(\pi_{C}^{*}(D)\right)^{\times}\right),
\end{aligned}
$$

where $U$ ranges over all open subschemes of $C^{N}$ containing $\pi_{C}^{*}(D)$.

One can then check as in the classical case (see for instance [Binda and Saito 2014, Theorem 3.3]) that there is a canonical isomorphism

$$
\frac{\mathcal{Z}_{0}(X, D)}{\mathcal{R}_{0}^{\prime}(X, D)} \stackrel{\sim}{\rightarrow} \mathrm{CH}_{0}(X, D)
$$

\section{The cycle class map}

Let $(X, D)$ be a 2-dimensional modulus pair. In this section, we construct the cycle class map $\mathrm{CH}_{0}(X, D) \rightarrow H^{2}\left(X, \mathcal{K}_{2,(X, D)}\right)$ and prove Theorems 1.2 and 1.4. More generally, we assume $X$ is either a curve or a surface and let $P \in X \backslash D$ be a closed point. Let $X_{P}$ denote the spectrum of the local ring $\mathcal{O}_{X, P}$. Assume $d=1,2$. It follows from (2-1) and (2-2) that there is a commutative diagram

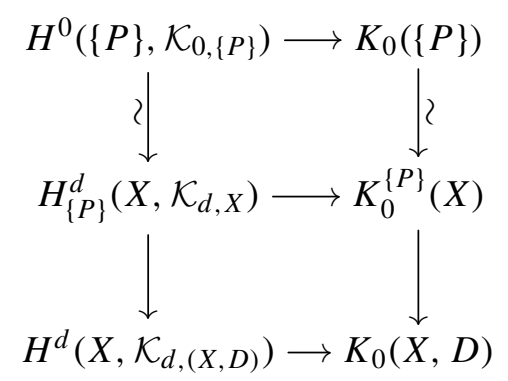

where the top vertical arrow on the left is an isomorphism by excision and the Gersten resolution for $\mathcal{K}_{d, X_{P}}$ and the one on the right is an isomorphism by the localization sequence for $K$-theory. We define the cycle class map

$$
\operatorname{cyc}_{(X, D)}: \mathcal{Z}_{0}(X, D) \longrightarrow H^{d}\left(X, \mathcal{K}_{d,(X, D)}\right)
$$


by letting $\operatorname{cyc}_{(X, D)}([P])$ be the image of $1 \in H^{0}\left(\{P\}, \mathcal{K}_{0,\{P\}}\right) \simeq \mathbb{Z}$ under the composite vertical arrow on the left in (3-1) and extending it linearly on all of $\mathcal{Z}_{0}(X, D)$. To show that this map kills rational equivalences, we first consider the case of curves.

Lemma 3.1. Let $(C, D)$ be an 1-dimensional modulus pair. Then the map $\mathrm{cyc}_{(C, D)}$ induces isomorphisms

$$
\begin{aligned}
& \operatorname{cyc}_{(C, D)}: \mathrm{CH}_{0}(C, D) \stackrel{\sim}{\longrightarrow} H_{\text {zar }}^{1}\left(C, \mathcal{K}_{1,(C, D)}\right) \\
& \stackrel{\sim}{\longrightarrow} H_{\text {nis }}^{1}\left(C, \mathcal{K}_{1,(C, D)}\right) \stackrel{\sim}{\longrightarrow} \operatorname{Pic}(C, D) \stackrel{\sim}{\longrightarrow} K_{0}(C, D) .
\end{aligned}
$$

Proof. For any reduced closed subset $S \subsetneq C$ such that $S \cap D=\varnothing$ and any open subset $U \subseteq X$, we have the localization fiber sequence of spectra

$$
K(S \cap U) \longrightarrow K(U) \longrightarrow K(U \backslash S) .
$$

Taking the filtered colimit over closed subsets $S$ as above under the inclusion, we get a short exact sequence of Zariski sheaves

$$
0 \longrightarrow \mathcal{K}_{1,(C, D)} \longrightarrow j_{*}\left(\mathcal{K}_{1,\left(C_{D}, D\right)}\right) \longrightarrow \coprod_{P \notin D}\left(i_{P}\right)_{*}\left(K_{0}(k(P))\right) \longrightarrow 0
$$

on $C$, where $C_{D}$ is the spectrum of the semilocal ring $A_{C \mid D}$ of $C$ at $|D|$ and $j: C_{D} \hookrightarrow C$ is the inclusion map. This yields the cycle class map

$$
\operatorname{cyc}_{(C, D)}: \coprod_{P \notin D} \mathbb{Z} \longrightarrow H^{1}\left(C, \mathcal{K}_{1,(C, D)}\right) .
$$

To show that this induces an isomorphism $\mathrm{CH}_{0}(C, D) \rightarrow H^{1}\left(C, \mathcal{K}_{1,(C, D)}\right)$, we first claim that $j_{*}\left(\mathcal{K}_{1,\left(C_{D}, D\right)}\right)$ is an acyclic Zariski sheaf. To prove this claim, it suffices to show that if $U \hookrightarrow C$ is open and $U_{D}$ is the spectrum of the semilocal ring of $U$ at $|U \cap D|$, then $H^{i}\left(U_{D}, \mathcal{K}_{1,\left(U_{D}, D\right)}\right)=0$ for $i \geq 1$. But this is immediate from the exact sequence

$$
0 \longrightarrow \mathcal{K}_{1,\left(U_{D}, D\right)} \longrightarrow \mathcal{K}_{1, U_{D}} \longrightarrow \mathcal{K}_{1, U \cap D} \longrightarrow 0
$$

and the fact that $U_{D}$ is a semilocal scheme.

It follows from the above claim that (3-3) is an acyclic resolution of $\mathcal{K}_{1,(C, D)}$ and in particular, there is an exact sequence

$$
K_{1}\left(A_{C \mid D}, I_{D}\right) \stackrel{\text { div }}{\longrightarrow} \coprod_{P \notin D} \mathbb{Z} \longrightarrow H_{\text {zar }}^{1}\left(C, \mathcal{K}_{1,(C, D)}\right) \longrightarrow 0 .
$$

By (2-8), this implies that the map (3-4) induces an isomorphism $\mathrm{CH}_{0}(C, D) \stackrel{\sim}{\longrightarrow}$ $H_{\text {zar }}^{1}\left(C, \mathcal{K}_{1,(C, D)}\right)$.

The isomorphism of the natural map $H_{\text {zar }}^{1}\left(C, \mathcal{K}_{1,(C, D)}\right) \rightarrow H_{\text {nis }}^{1}\left(C, \mathcal{K}_{1,(C, D)}\right)$ follows easily from Hilbert's theorem 90. 
We now consider the commutative diagram of homotopy fiber sequences

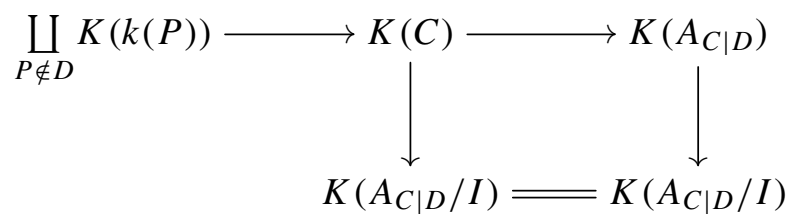

This yields a homotopy fiber sequence

$$
\coprod_{P \notin D} K(k(P)) \longrightarrow K(C, D) \longrightarrow K\left(A_{C \mid D}, I\right)
$$

and in particular, an exact sequence

$$
K_{1}\left(A_{C \mid D}, I\right) \stackrel{\partial}{\longrightarrow} \mathcal{Z}_{0}(C, D) \longrightarrow K_{0}(C, D) \longrightarrow 0
$$

and we conclude from this that

$$
\operatorname{Coker}(\partial)=\mathrm{CH}_{0}(C, D) \stackrel{\sim}{\longrightarrow} K_{0}(C, D) .
$$

Finally, the isomorphism $H_{\text {zar }}^{1}\left(C, \mathcal{K}_{1,(C, D)}\right) \stackrel{\sim}{\longrightarrow} \operatorname{Pic}(C, D)$ follows from [Suslin and Voevodsky 1996, Lemma 2.1].

Lemma 3.2. Let $(X, D)$ be a 2-dimensional modulus pair and let $f: C \rightarrow X$ be a finite map, where $C$ is a nonsingular curve such that $f^{*}(D)$ is a proper closed subscheme of $C$. Then there is a commutative diagram

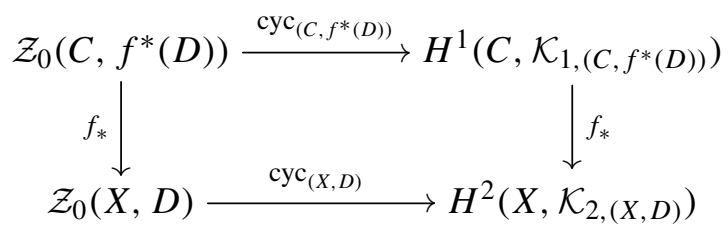

where $f_{*}$ on the left is the pushforward map.

Proof. We set $E=f^{*}(D)$. Since $\iota_{X}: D \hookrightarrow X$ and $\iota_{C}: E \hookrightarrow C$ are Cartier divisors, $\operatorname{Tor}_{\mathcal{O}_{X}}^{i}\left(\mathcal{O}_{D}, f_{*}\left(\mathcal{O}_{C}\right)\right)=0$ for $i>0$. In particular, there is a commutative diagram

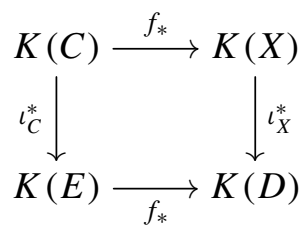

As (3-6) makes sense for any open $U \hookrightarrow X$ and is functorial for restriction to open subsets, we see that it is in fact a diagram of presheaves of spectra on $X_{\mathrm{zar}}$.

If we consider the homotopy cofibers of the horizontal arrows in (3-6), we obtain a commutative diagram of homotopy cofiber sequences of presheaves of spectra 
on $X_{\text {zar }}$. Taking the long homotopy groups exact sequences, we obtain the associated diagram of the long exact sequences of the presheaves of homotopy groups. The exactness of the sheafification functor yields a commutative diagram of the long exact sequences of the sheaves of homotopy groups corresponding to (3-6).

Let $\widetilde{K}(X \backslash C)$ and $\widetilde{K}(D \backslash E)$ denote the homotopy cofibers of the top and bottom horizontal arrows in (3-6), respectively. Let $\widetilde{\mathcal{K}}_{i, X \backslash C}$ denote the Zariski sheaf on $X$ associated to the presheaf of homotopy groups $U \mapsto \pi_{i}(\widetilde{K}(U \backslash C))$. Defining $\widetilde{\mathcal{K}}_{i, D \backslash E}$ in a similar way, we get a commutative diagram of the long exact sequences

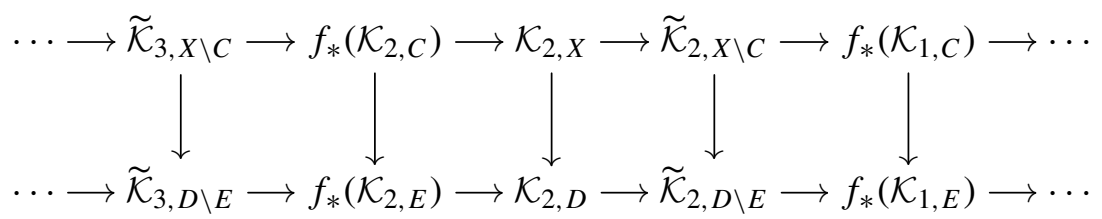

If $\bar{C}$ is the image of $f: C \rightarrow X$, then we have a factorization $K(C) \rightarrow G(\bar{C}) \rightarrow$ $K(X)$ (see [Srinivas 1991, Proposition 5.12(i)]) and this shows that there is a factorization $\mathcal{K}_{i, X} \rightarrow \widetilde{\mathcal{K}}_{i, X \backslash C} \rightarrow j_{*}\left(\mathcal{K}_{i, X \backslash \bar{C}}\right) \rightarrow j_{*}\left(K_{i}(k(X))\right)$, where $j: X \backslash \bar{C} \hookrightarrow X$ is the inclusion. The Gersten resolution says that the composite map is injective. Hence, the map $\mathcal{K}_{i, X} \rightarrow \widetilde{\mathcal{K}}_{i, X \backslash C}$ is injective. Since the map $f_{*}\left(\mathcal{K}_{i, C}\right) \rightarrow f_{*}\left(\mathcal{K}_{i, E}\right)$ is surjective for $i \leq 2$, the above diagram refines to a commutative diagram of short exact sequences

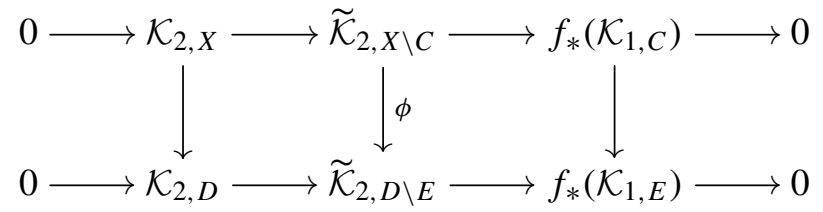

Set $\widetilde{\mathcal{K}}_{2,(X, D)}=\operatorname{Ker}\left(\mathcal{K}_{2, X} \rightarrow \mathcal{K}_{2, D}\right)$. Since the vertical arrows on the left and the right ends in (3-8) are surjective, the middle arrow is also surjective and there is a short exact sequence of the kernel sheaves

$$
0 \longrightarrow \widetilde{\mathcal{K}}_{2,(X, D)} \longrightarrow \operatorname{Ker}(\phi) \longrightarrow f_{*}\left(\mathcal{K}_{1,(C, E)}\right) \longrightarrow 0 .
$$

Considering the long exact cohomology sequences with and without support and observing that $H^{i}\left(C, f_{*}\left(\mathcal{K}_{1,(C, E)}\right)\right) \simeq H^{i}\left(C, \mathcal{K}_{1,(C, E)}\right)$ (the higher direct images of $\mathcal{K}_{1,(C, E)}$ vanish as one can easily check), we get a commutative diagram

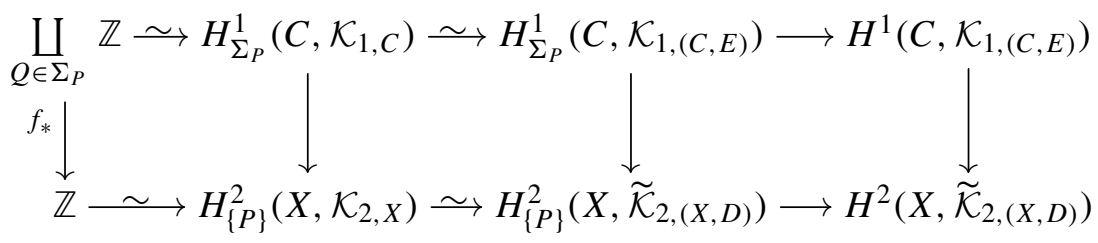


for any closed point $P \in X \backslash D$ and $\Sigma_{P}=f^{-1}(P)$. It is well known that the leftmost vertical map is the pushforward map. Since the map $\mathcal{K}_{2,(X, D)} \rightarrow \widetilde{\mathcal{K}}_{2,(X, D)}$ is a surjective map whose kernel is supported on $D$, the map $H^{2}\left(X, \mathcal{K}_{2,(X, D)}\right) \rightarrow$ $H^{2}\left(X, \widetilde{\mathcal{K}}_{2,(X, D)}\right)$ is an isomorphism. This immediately yields (3-5).

Proof of Theorem 1.2. In view of Lemma 2.1, the proof of Theorem 1.2 is reduced to showing that the cycle class map $\operatorname{cyc}_{(X, D)}: \mathcal{Z}_{0}(X, D) \rightarrow H^{2}\left(X, \mathcal{K}_{2,(X, D)}\right)$ constructed in (3-2) kills the group of rational equivalences $\mathcal{R}_{0}^{\prime}(X, D)$ (see (2-8)) and is surjective. So, let us take an integral curve $C \hookrightarrow X$ which is not contained in $D$ and let $f: C^{N} \rightarrow X$ denote the induced map from the normalization of $C$. Letting $E=f^{*}(D)$ and $g \in \operatorname{Ker}\left(\mathcal{O}_{C^{N}}^{\times} \rightarrow \mathcal{O}_{E}^{\times}\right)$, we need to show that $\operatorname{cyc}_{(X, D)} \circ f_{*}(\operatorname{div}(g))=0$. For this, we consider the diagram

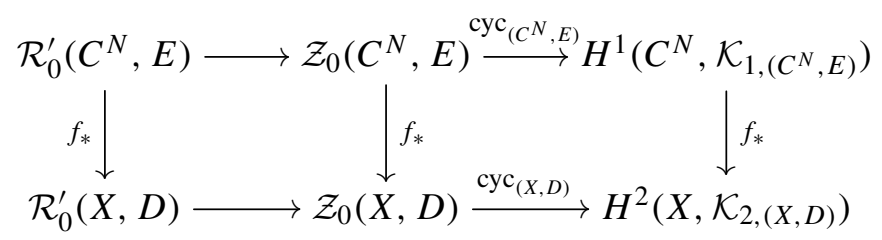

in which the left square commutes by [Krishna and Park 2014, Proposition 2.10] and the right square commutes by Lemma 3.2. Since the composite horizontal map on the top is zero by Lemma 3.1, it follows that

$$
\operatorname{cyc}_{(X, D)} \circ f_{*}(\operatorname{div}(g))=f_{*} \circ \operatorname{cyc}_{\left(C^{N}, E\right)}(\operatorname{div}(g))=0 .
$$

The surjectivity of $\operatorname{cyc}_{(X, D)}$ now follows from Lemma 3.2, the isomorphism $\mathcal{K}_{2,(X, D)}^{M} \stackrel{\sim}{\rightarrow} \widetilde{\mathcal{K}}_{2,(X, D)}$, the diagram (3-1) and [Kato and Saito 1986, Theorem 2.5].

Proof of Theorem 1.4. Let $\pi: X \rightarrow Y$ be a resolution of singularities of a normal surface over any field $k$. We set $U=Y_{\text {reg }}$ and $C(U)=\lim _{D} \mathrm{CH}_{0}(X, D)$, where the limit is taken over all effective Cartier divisors on $X$ with support outside $U$. Let $E \hookrightarrow X$ denote the reduced exceptional divisor. If $D \subsetneq X$ is an effective Cartier divisor with support $|D| \subseteq E$, then $m E-D$ must be an effective Cartier divisor some $m \gg 1$. This implies that the canonical maps

$C(U) \rightarrow \underset{m}{\lim } \mathrm{CH}_{0}(X, m E) \quad$ and ${\underset{\lim }{\leftrightarrows}}_{D} H^{2}\left(X, \mathcal{K}_{2,(X, D)}\right) \rightarrow \underset{m}{\lim } H^{2}\left(X, \mathcal{K}_{2,(X, m E)}\right)$ are isomorphisms.

Let $\mathrm{CH}_{0}(Y)$ denote the Chow group of 0-cycles on $Y$ in the sense of [Levine and Weibel 1985] and let $S \hookrightarrow Y$ denote the singular locus of $Y$ with reduced 
subscheme structure. We then have a commutative diagram

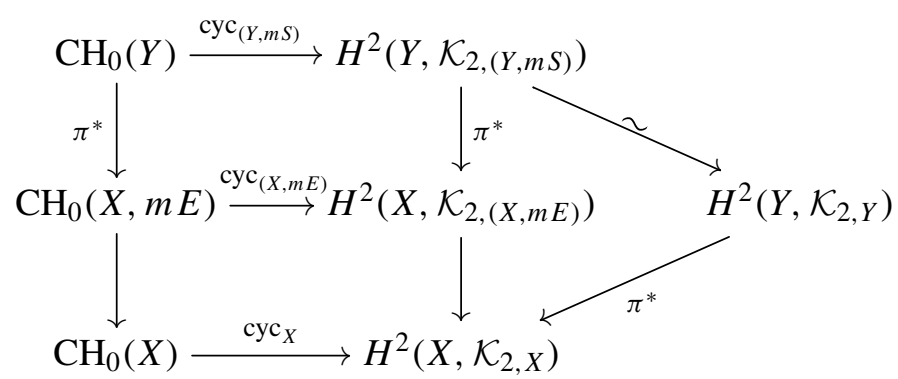

The map cyc $\mathrm{c}_{(Y, m S)}$ is defined exactly like $\operatorname{cyc}_{(X, m E)}$ and is an isomorphism by [Krishna 2015, Proposition 3.1]. The natural map $H^{2}\left(Y, \mathcal{K}_{2,(Y, m S)}\right) \rightarrow H^{2}\left(Y, \mathcal{K}_{2, Y}\right)$ is an isomorphism also by [Krishna 2015, Proposition 3.1]. The map $\pi^{*}: \mathrm{CH}_{0}(Y) \rightarrow$ $\mathrm{CH}_{0}(X, m E)$ is induced by the identity map $\pi^{*}: \mathcal{Z}_{0}(U) \rightarrow \mathcal{Z}_{0}(X, m E)$.

To show that it preserves rational equivalences, let $C \hookrightarrow Y$ be an integral curve not meeting $S$ and let $h \in k(C)^{\times}$. Let $\Gamma_{h} \hookrightarrow C \times \mathbb{P}^{1} \hookrightarrow Y \times \mathbb{P}^{1}$ be the graph of the function $h: C \rightarrow \mathbb{P}^{1}$. It is then clear that $\Gamma_{h} \cap\left(S \times \mathbb{P}^{1}\right)=\varnothing$. In particular, $\pi^{-1}\left(\Gamma_{h}\right) \cap\left(E \times \mathbb{P}^{1}\right)=\varnothing$. This shows that $\left[\Gamma_{h}\right] \in \mathcal{Z}_{1}(X, m E)$ is an admissible 1 -cycle such that

$\pi^{*}(\operatorname{div}(h))=\pi^{*}\left(\left[h^{*}(0)\right]-\left[h^{*}(\infty)\right]\right)=\pi^{*}\left(\partial_{0}\left(\left[\Gamma_{h}\right]\right)-\partial_{\infty}\left(\left[\Gamma_{h}\right]\right)\right)=\left(\partial_{0}-\partial_{\infty}\right)\left(\left[\Gamma_{h}\right]\right)$.

This shows the inclusion $\pi^{*}(\operatorname{div}(h)) \subset \mathcal{R}_{0}(X, m E)$ and it yields the pullback $\pi^{*}: \mathrm{CH}_{0}(Y) \rightarrow \mathrm{CH}_{0}(X, m E)$. All other maps in (3-12) are naturally defined and all are surjective.

If we let $F^{2} K_{0}(X, m E)$ denote the image of the map cyc $(X, m E): \mathrm{CH}_{0}(X, m E) \rightarrow$ $K_{0}(X, m E)$, then it follows from Theorem 1.2 and Lemma 2.1 that $F^{2} K_{0}(X, m E) \rightarrow$ $H^{2}\left(X, \mathcal{K}_{2,(X, m E)}\right)$ is an isomorphism. We now apply [Krishna and Srinivas 2002, Theorem 1.1] to conclude that the map $H^{2}\left(Y, \mathcal{K}_{2,(Y, m S)}\right) \rightarrow H^{2}\left(X, \mathcal{K}_{2,(X, m E)}\right)$ is an isomorphism for all sufficiently large $m$. It follows that all arrows in the upper square of (3-12) are isomorphisms for all sufficiently large $m$. In particular, the map $\operatorname{cyc}_{(X, m E)}: \mathrm{CH}_{0}(X, m E) \rightarrow H^{2}\left(X, \mathcal{K}_{2,(X, m E)}\right)$ is an isomorphism for all sufficiently large $m$ and hence the map $C(U) \rightarrow \underset{m}{\lim } H^{2}\left(X, \mathcal{K}_{2,(X, m E)}\right)$ is an isomorphism.

\section{Vanishing theorems and failure of localization}

Let $k$ be a field and consider the effective Cartier divisor $D=\operatorname{Spec}\left(k[t] / t^{m}\right)$ on $\mathbb{A}_{k}^{1}=\operatorname{Spec}(k[t])$. Given $X \in \mathbf{S c h} / k$, let us denote the effective Cartier divisor $X \times D \hookrightarrow X \times \mathbb{A}_{k}^{1}$ by $D$ itself. We shall prove Theorem 1.6 using the following algebraic result. 
Lemma 4.1. Let $A$ be the coordinate ring of a smooth affine curve over $k$ and let $\mathfrak{m}$ be a maximal ideal of $A[t]$ which contains the ideal $(t-a)$, where $a \in k^{\times}$. Then we can find a prime ideal $\mathfrak{p}$ of height one in $A[t]$ such that the following hold.

(1) $\mathfrak{p} \subsetneq \mathfrak{m}$.

(2) $A[t] / \mathfrak{p}$ is smooth.

(3) $\mathfrak{m} / \mathfrak{p}$ is a principal ideal.

(4) $\mathfrak{p}+(t)=A[t]$.

Proof. Consider the maximal ideal $\mathfrak{m}^{\prime}=\mathfrak{m} \cap A$ of $A$. Since $A$ is a Dedekind domain, we can write $\mathfrak{m}^{\prime}=\left(f_{1}, f_{2}\right)$. But this implies using our hypothesis that $\mathfrak{m}=\left(t-a, f_{1}, f_{2}\right)=\left(a^{-1} t-1, f_{1}, f_{2}\right)$. In case $f_{1}=f_{2}$, we take $\mathfrak{p}=(t-a)$ which clearly does the job. So we assume that $f_{1} \neq f_{2}$.

Since $A_{\mathfrak{m}^{\prime}}$ is a discrete valuation ring, $\mathfrak{m}^{\prime} A_{\mathfrak{m}^{\prime}}$ is a principal ideal. In particular, there is an element $f \in A$ such that $f \notin \mathfrak{m}^{\prime}$ and $\mathfrak{m}^{\prime} A_{f}$ is principal. As $f \notin \mathfrak{m}^{\prime}$, we have $(f)+\mathfrak{m}^{\prime}=A$, and this gives us an identity $\alpha f-\alpha_{1} f_{1}-\alpha_{2} f_{2}-1=0$ in $A$. Setting $g=\alpha f$, we see that $\mathfrak{m}^{\prime} A_{g}$ is also a principal ideal. Furthermore, we have

$$
g a^{-1} t-1=g\left(a^{-1} t-1\right)+g-1=g\left(a^{-1} t-1\right)+\alpha_{1} f_{1}+\alpha_{2} f_{2} \in \mathfrak{m} .
$$

If we set $\mathfrak{p}=\left(g a^{-1} t-1\right) \subsetneq A[t]$, we have just shown that $\mathfrak{p} \subsetneq \mathfrak{m}$. Since $A[t] / \mathfrak{p} \simeq A_{g}$ and hence

$$
\frac{\mathfrak{m}}{\mathfrak{p}} \simeq \frac{\mathfrak{m} A_{g}[t]}{\mathfrak{p} A_{g}[t]} \simeq \frac{\left(-g^{-1}\left(\alpha_{1} f_{1}+\alpha_{2} f_{2}\right), f_{1}, f_{2}\right) A_{g}[t]}{\mathfrak{p} A_{g}[t]} \simeq \frac{\left(f_{1}, f_{2}\right) A_{g}[t]}{\mathfrak{p} A_{g}[t]} \simeq \mathfrak{m}^{\prime} A_{g},
$$

we see that (2) and (3) are satisfied. The item (4) is clear. This proves the lemma.

Proof of Theorem 1.6. We can assume that $Y$ is connected. We set $X=Y \times \mathbb{A}_{k}^{1}$ and $U=Y \times \mathbb{G}_{m}$. Let $p: X \rightarrow \mathbb{A}_{k}^{1}$ and $q: X \rightarrow Y$ denote the projection maps. Let $P \in U$ be a closed point and set $P_{1}=p(P)$ and $P_{2}=q(P)$. Then $P_{1} \in \mathbb{G}_{m}$ and $P_{2} \in Y$ are closed points as well.

We can find a nonsingular curve $\iota: C \hookrightarrow Y$ containing $P_{2}$ (see [Kleiman and Altman 1979, Theorem 1] when $k$ is infinite and [Poonen 2008, Theorem 1.1] when $k$ is finite). It follows from [Krishna and Park 2014, Proposition 2.10] that there is a pushforward map $\iota_{*}: \mathrm{CH}_{0}\left(C \times \mathbb{A}_{k}^{1}, D\right) \rightarrow \mathrm{CH}_{0}\left(Y \times \mathbb{A}_{k}^{1}, D\right)$ such that the class $[P] \in \mathrm{CH}_{0}\left(Y \times \mathbb{A}_{k}^{1}, D\right)$ lies in the image of this map. We can therefore assume that $Y$ is a curve.

Now $P$ defines a unique closed point $P^{\prime} \in X_{k(P)}$ such that $P=\pi\left(P^{\prime}\right)$, where $\pi: \operatorname{Spec}(k(P)) \rightarrow \operatorname{Spec}(k)$ is the finite map. This gives $[P]=\pi_{*}\left(\left[P^{\prime}\right]\right)$ under the pushforward map $\pi_{*}: \mathrm{CH}_{0}\left(X_{k(P)}, D\right) \rightarrow \mathrm{CH}_{0}(X, D)$ (see [Krishna and Park 2014, Proposition 2.10]). It suffices therefore to show that the class $\left[P^{\prime}\right] \in \mathrm{CH}_{0}\left(X_{k(P)}, D\right)$ dies. We can thus assume that $P_{1} \in \mathbb{G}_{m}(k)$. 
We can now apply Lemma 4.1 to get a smooth affine curve $i: C \hookrightarrow X$ which is a closed subset of $X$ containing $P$ such that $C \cap(Y \times D)=\varnothing$ and $P \in C$ is a principal Cartier divisor. In particular, the class $[P] \in \mathrm{CH}_{0}(C)$ is zero. On the other hand, the condition $C \cap(Y \times D)=\varnothing$ implies that the inclusion $\mathcal{Z}_{0}(C) \hookrightarrow \mathcal{Z}_{0}(X, D)$ defines a pushforward map $i_{*}: \mathrm{CH}_{0}(C) \rightarrow \mathrm{CH}_{0}(X, D)$ (see [Krishna and Park 2014, Corollary 2.11]) such that $i_{*}([P])=[P] \in \mathrm{CH}_{0}(X, D)$. It follows that $[P]=0$. This proves that $\mathrm{CH}_{0}(X, D)=0$. The second part of the theorem now follows from Theorem 1.2.

As an immediate consequence of Theorems 1.2 and 1.6, we get:

Corollary 4.2. Given a nonsingular affine curve $Y$ over a field $k$, we have

$$
K_{0}\left(Y \times \mathbb{A}_{k}^{1}, D\right) \stackrel{\sim}{\longrightarrow} \operatorname{Pic}\left(Y \times \mathbb{A}_{k}^{1}, D\right) .
$$

Remark 4.3. Theorem 1.6 is known to fail when $d=0$ (see [Bloch and Esnault 2003a]).

Proof of Theorem 1.7. Let the pair $(X, D)$ be as in Theorem 1.7 and let $x \in X \backslash D$ be a closed point. We can assume that $X$ is connected. We claim that there is a smooth affine closed subscheme $\iota: Y \hookrightarrow X$ of dimension $d-1$ such that $Y \cap D=\varnothing$ and $x \in Y$.

To prove the claim, let $A$ denote the coordinate ring of $X$ and let $I \hookrightarrow A$ denote the defining ideal of $D$. Let $\mathfrak{m} \hookrightarrow A$ denote the maximal ideal corresponding to $x \in X$. Our assumption implies that there exist elements $a \in \mathfrak{m}^{2}$ and $b \in I$ such that $a-b=1$. We can now apply [Swan 1974, Theorems 1.3,1.4] to conclude that for general $a^{\prime} \in \mathfrak{m}^{2}$, the ring $A /\left(a-a^{\prime} b\right)$ is integral and smooth. Setting $f=a-a^{\prime} b$, we see that $f \in \mathfrak{m}$ and $f-1=a-a^{\prime} b-1=b-a^{\prime} b=b\left(1-a^{\prime}\right) \in I$. This shows that $Y:=\operatorname{Spec}(A /(f))$ satisfies our requirement.

Using the above claim and [Krishna and Park 2014, Corollary 2.11], we get a pushforward map $\iota_{*}: \mathrm{CH}_{0}(Y) \rightarrow \mathrm{CH}_{0}(X, D)$ whose image contains the cycle class $[x]$. The desired vanishing now follows because one knows that $\mathrm{CH}_{0}(Y)=0$ (see for instance [Krishna and Srinivas 2007, Theorem 6.4.1]).

To prove the second assertion of the theorem, we first notice that for a closed point $x \in X \backslash D$, we have natural maps

$$
K_{0}(k(x)) \stackrel{\sim}{\rightarrow} H_{\{x\}}^{d}\left(X, \mathcal{K}_{d,(X, D)}^{M}\right) \longrightarrow H_{\text {zar }}^{d}\left(X, \mathcal{K}_{d,(X, D)}^{M}\right) \longrightarrow H_{\text {nis }}^{d}\left(X, \mathcal{K}_{d,(X, D)}^{M}\right) .
$$

Setting $\operatorname{cyc}_{(X, D)}([x])$ to be the image of $1 \in K_{0}(k(x))$ under the composite map, we get a cycle class map $\operatorname{cyc}_{(X, D)}: \mathcal{Z}_{0}(X, D) \rightarrow H_{\text {nis }}^{d}\left(X, \mathcal{K}_{d,(X, D)}^{M}\right)$.

If $D_{\text {red }}$ has normal crossings, then it follows from [Rülling and Saito 2015, Definition 3.4.1, Proposition 3.5] that $\operatorname{cyc}_{(X, D)}$ has a factorization $\mathrm{CH}_{0}(X, D) \rightarrow$ $\mathbb{U}_{\text {nis }}^{2 d}\left(X, \mathcal{Z}(d)_{X \mid D}\right) \rightarrow H_{\text {nis }}^{d}\left(X, \mathcal{K}_{d,(X, D)}^{M}\right)$, where $\mathcal{Z}(d)_{X \mid D}$ is the sheaf of cycle complexes $U \mapsto \mathcal{Z}^{d}(U \mid D, 2 d-\bullet)$ on $X_{\text {nis }}$. Moreover, it follows from [Kato and Saito 
1986, Theorem 2.5] that the map $\operatorname{cyc}_{(X, D)}: \mathrm{CH}_{0}(X, D) \rightarrow H_{\text {nis }}^{d}\left(X, \mathcal{K}_{d,(X, D)}^{M}\right)$ is surjective. The vanishing of $H_{\text {nis }}^{d}\left(X, \mathcal{K}_{d,(X, D)}^{M}\right)$ now follows from the first part of the theorem.

Proof of Theorem 1.5. In view of Theorem 1.6, the theorem is equivalent to the assertion that the pushforward map $\mathrm{CH}_{0}\left(\{P\} \times \mathbb{A}_{k}^{1}, D\right) \stackrel{i_{*}}{\longrightarrow} \mathrm{CH}_{0}\left(Y \times \mathbb{A}_{k}^{1}, D\right)$ is not surjective. If we let $\pi: Y \rightarrow \operatorname{Spec}(k)$ denote the structure map, then the composite map $\mathrm{CH}_{0}\left(\{P\} \times \mathbb{A}_{k}^{1}, D\right) \stackrel{i_{*}}{\longrightarrow} \mathrm{CH}_{0}\left(Y \times \mathbb{A}_{k}^{1}, D\right) \stackrel{\pi_{*}}{\longrightarrow} \mathrm{CH}_{0}\left(\mathbb{A}_{k}^{1}, D\right)$ is an isomorphism. In particular, $i_{*}$ is split injective. Our aim is to show that it is not surjective.

We set $X=Y \times \mathbb{A}_{k}^{1}, V=Y \backslash\{P\}, U=V \times \mathbb{A}_{k}^{1}$ and $Z=\{P\} \times \mathbb{A}_{k}^{1}$. For any $W \in \mathbf{S c h} / k$, we shall write $W \times D$ as $W_{D}$ in this proof. In view of Theorem 1.2, it suffices to show that the composite map $\mathrm{CH}_{0}(Z, D) \stackrel{i_{*}}{\longrightarrow} \mathrm{CH}_{0}(X, D) \stackrel{\mathrm{cyc}_{(X, D)}}{\longrightarrow}$ $H^{2}\left(X, \mathcal{K}_{2,(X, D)}\right)$ is not surjective.

Let $\mathcal{H}_{Y_{D}}^{P}$ denote the exact category of coherent sheaves on $Y_{D}$ which have cohomological dimension at most one and which are supported on $\{P\} \times D$ so that there is a commutative diagram of the fiber sequences of spectra (see [Srinivas 1991, Theorem 9.1])

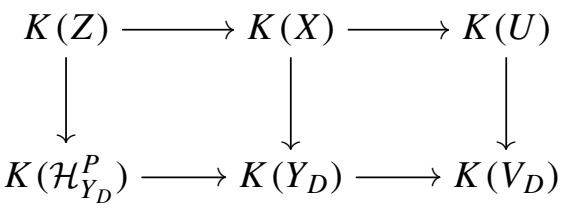

As in the proof of Lemma 3.2, this diagram canonically extends to a commutative diagram of presheaves of spectra. Let $\mathcal{K}_{i, Y_{D}}^{P}$ denote the Zariski sheaf on $Z$ associated to the presheaf of homotopy groups $W \mapsto \pi_{i}\left(K\left(\mathcal{H}_{Y_{D} \cap W}^{P}\right)\right)$. Sheafifying the associated presheaves of homotopy groups and arguing as in the proof of Lemma 3.2, we obtain the commutative diagrams of short exact sequence of Zariski sheaves

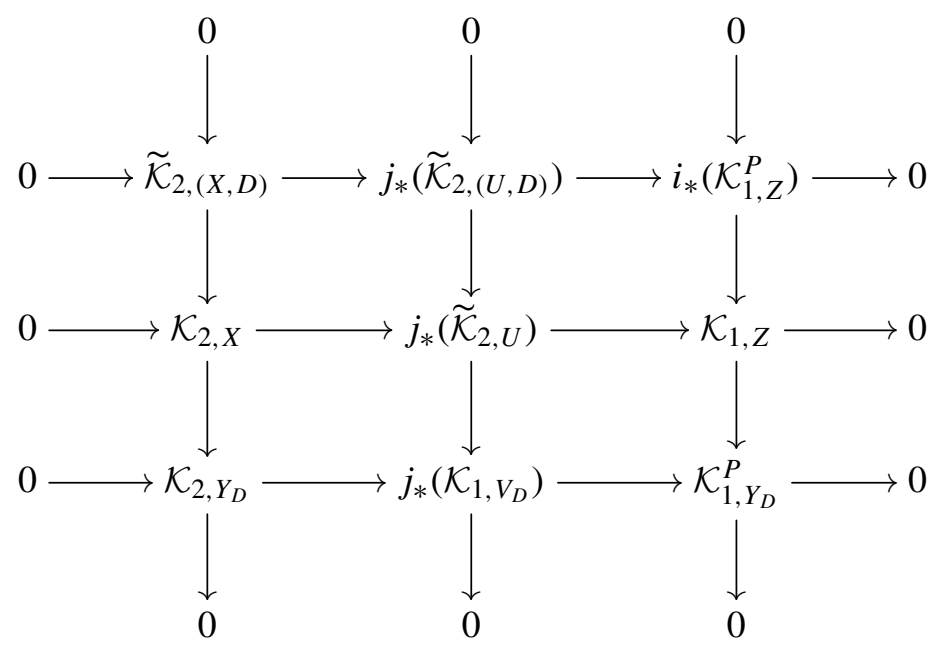


and

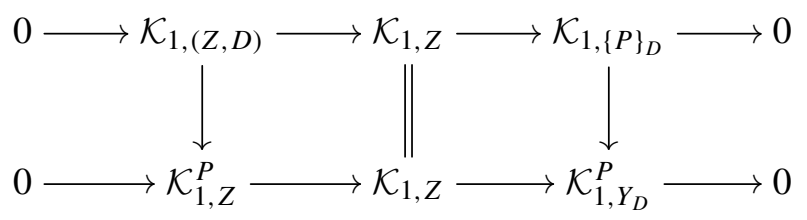

These diagrams together give rise to a commutative diagram of exact sequences

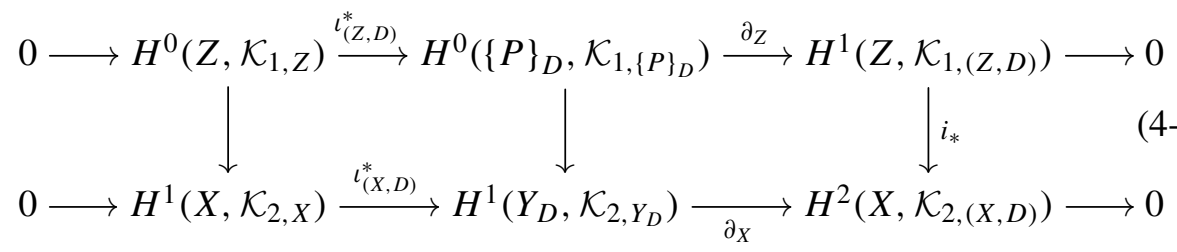

The maps $\partial_{Z}$ and $\partial_{X}$ are surjective because $H^{1}\left(Z, \mathcal{K}_{1, Z}\right) \simeq \mathrm{CH}_{0}(Z)=0=$ $\mathrm{CH}_{2}(X) \simeq H^{2}\left(X, \mathcal{K}_{2}, X\right)$. By the homotopy invariance of $K$-theory, the composite map $H^{0}\left(Z, \mathcal{K}_{1, Z}\right) \stackrel{l_{(Z, D)}^{*}}{\longrightarrow} H^{0}\left(\{P\}_{D}, \mathcal{K}_{\left.1,\{P\}_{D}\right)}\right) \longrightarrow H^{0}\left(\{P\}, \mathcal{K}_{1,\{P\}}\right)$ is an isomorphism. We claim that the composite map $H^{1}\left(X, \mathcal{K}_{2, X}\right) \stackrel{\iota_{(X, D)}^{*}}{\longrightarrow} H^{1}\left(Y_{D}, \mathcal{K}_{2, Y_{D}}\right) \longrightarrow$ $H^{1}\left(Y, \mathcal{K}_{2, Y}\right)$ is also an isomorphism.

We have a commutative diagram

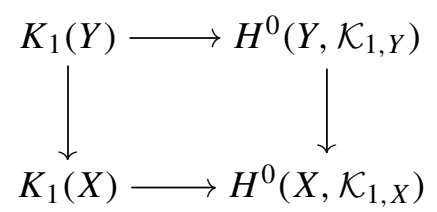

where the vertical arrows are isomorphisms and the horizontal arrows are split surjections. This implies that the induced pullback map $S K_{1}(Y) \rightarrow S K_{1}(X)$ is an isomorphism. We now have a commutative diagram

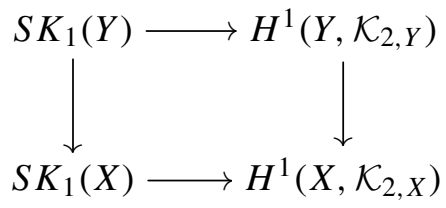

where the top horizontal arrow is an isomorphism and the bottom horizontal arrow is surjective (see [Krishna and Srinivas 2002, Lemma 2.3]). We have shown above that the left vertical arrow is an isomorphism. This implies that the right vertical arrow is surjective. On the other hand, it is split injective via the 0 -section embedding. Hence, it is an isomorphism. This proves the claim. 
The claim shows that the first horizontal arrows from left in both rows of (4-5) are split injective. Combining this with Lemmas 3.1 and 3.2, we can identify $i_{*}: \mathrm{CH}_{0}(Z, D) \rightarrow H^{2}\left(X, \mathcal{K}_{2,(X, D)}\right)$ as the map

$$
i_{*}: K_{1}(\{P\} \times D,\{P\} \times\{0\}) \rightarrow H^{1}\left(Y_{D}, \mathcal{K}_{2,\left(Y_{D}, Y\right)}\right) .
$$

Using [Krishna and Srinivas 2002, Corollary 4.2], this map is same as the map of $\mathbb{Q}$-vector spaces

$$
i_{*}: I \rightarrow H^{1}\left(Y_{D}, \frac{\Omega_{\left(Y_{D}, Y\right) / \mathbb{Q}}^{1}}{d\left(I_{Y}\right)}\right),
$$

where $I$ is the ideal sheaf of $\operatorname{Spec}(k)$ inside $D, I_{Y}=I \otimes_{k} \mathcal{O}_{Y}$ and $\Omega_{\left(Y_{D}, Y\right) / \mathbb{Q}}^{1}=$ $\operatorname{Ker}\left(\Omega_{Y_{D} / \mathbb{Q}}^{1} \rightarrow \Omega_{Y / \mathbb{Q}}^{1}\right)$. We are thus reduced to showing that this map of $\mathbb{Q}$-vector spaces is not surjective. Notice that the assumption $m \geq 2$ implies that $I \neq 0$.

By [Krishna and Srinivas 2002, Lemma 4.3], there is a short exact sequence

$$
0 \longrightarrow \Omega_{k / \mathbb{Q}}^{1} \otimes_{k} I_{Y} \longrightarrow \frac{\Omega_{\left(Y_{D}, Y\right) / \mathbb{Q}}^{1}}{d\left(I_{Y}\right)} \longrightarrow \frac{\Omega_{\left(Y_{D}, Y\right) / k}^{1}}{d_{k}\left(I_{Y}\right)} \longrightarrow 0 .
$$

It is easy to check by local calculations that $\frac{\Omega_{\left(Y_{D}, Y\right) / k}^{1}}{d\left(I_{Y}\right)} \simeq \Omega_{Y / \mathbb{Q}}^{1} \otimes_{k} d_{k}(I)$, where $d_{k}: I \rightarrow \Omega_{D / k}^{1}$ is the $k$-derivation. In particular, the above sequence can be written as

$$
0 \rightarrow\left(I \otimes_{k} \Omega_{k / \mathbb{Q}}^{1}\right) \otimes_{k} \mathcal{O}_{Y} \rightarrow \mathcal{K}_{2,\left(Y_{D}, Y\right)} \rightarrow d_{k}(I) \otimes_{k} \Omega_{Y / k}^{1} \rightarrow 0 .
$$

Taking the associated long exact cohomology sequence, we get a commutative diagram

$$
\begin{aligned}
d_{k}(I) \otimes_{k} H^{0}\left(Y, \Omega_{Y / k}^{1}\right) \stackrel{\partial}{\rightarrow}\left(I \otimes_{k} \Omega_{k / \mathbb{Q}}^{1}\right) \otimes_{k} & H^{1}\left(Y, \mathcal{O}_{Y}\right) \\
& \rightarrow H^{1}\left(Y_{D}, \mathcal{K}_{2,\left(Y_{D}, Y\right)} \longrightarrow d_{k}(I) \rightarrow 0\right.
\end{aligned}
$$

with the top sequence exact.

It is straightforward to check that $d_{k}$ is an isomorphism. On the other hand, as $k$ has infinite transcendence degree over $\mathbb{Q}$ and $Y$ has positive genus, we see that $\partial$ is a map of $k$-vector spaces whose source is finite dimensional but the target is infinite dimensional. This shows that there is a split exact sequence

$$
0 \rightarrow \frac{\left(I \otimes_{k} \Omega_{k / \mathbb{Q}}^{1}\right) \otimes_{k} H^{1}\left(Y, \mathcal{O}_{Y}\right)}{d_{k}(I) \otimes_{k} H^{0}\left(Y, \Omega_{Y / k}^{1}\right)} \rightarrow H^{1}\left(Y_{D}, \mathcal{K}_{2,\left(Y_{D}, Y\right)}\right) \rightarrow d_{k}(I) \rightarrow 0
$$

such that the first term is an infinite dimensional $k$-vector space and the composite map $I \stackrel{i_{*}}{\longrightarrow} H^{1}\left(Y_{D}, \mathcal{K}_{2,\left(Y_{D}, Y\right)}\right) \rightarrow d_{k}(I)$ is an isomorphism. In particular, the cokernel of $i_{*}$ is an infinite dimensional $k$-vector space. This finishes the proof of Theorem 1.5. 


\section{Acknowledgements}

The author would like to thank Jinhyun Park for his questions related to connections between additive Chow groups and reciprocity functors that led to the section on page 2399. The author would like to thank Marc Levine and Federico Binda for invitation to the university of Duisburg-Essen at Essen in April 2015, where this paper was revised. The author would also like to thank the referee for carefully reading the paper and suggesting valuable improvements.

\section{References}

[Binda and Krishna $\geq 2015$ ] F. Binda and A. Krishna, "0-cycles with modulus", in preparation.

[Binda and Saito 2014] F. Binda and S. Saito, "Relative cycles with moduli and regulator maps", preprint, 2014. arXiv 1412.0385

[Bloch and Esnault 2003a] S. Bloch and H. Esnault, "The additive dilogarithm", Doc. Math. Extra Vol. (2003), 131-155. MR 2005e:19006 Zbl 1052.11048

[Bloch and Esnault 2003b] S. Bloch and H. Esnault, "An additive version of higher Chow groups", Ann. Sci. École Norm. Sup. (4) 36:3 (2003), 463-477. MR 2004c:14035 Zbl 1100.14014

[Fulton 1998] W. Fulton, Intersection theory, 2nd ed., Springer, Berlin, 1998. MR 99d:14003 Zbl 0885.14002

[Ivorra and Rülling $\geq 2015$ ] F. Ivorra and K. Rülling, " $K$-groups of reciprocity functors", preprint. To appear in J. Algebr. Geom. arXiv 1209.1217

[Kato and Saito 1986] K. Kato and S. Saito, "Global class field theory of arithmetic schemes", pp. 255-331 in Applications of algebraic K-theory to algebraic geometry and number theory, Part I, II (Boulder, CO, 1983), edited by S. J. Bloch et al., Contemp. Math. 55, Amer. Math. Soc., Providence, RI, 1986. MR 88c:11041 Zbl 0614.14001

[Kerz and Saito 2015] M. Kerz and S. Saito, "Chow group of 0-cycles with modulus and higher dimensional class field theory", preprint, 2015. To appear in Duke J. Math. arXiv 1304.4400v4

[Kleiman and Altman 1979] S. L. Kleiman and A. B. Altman, "Bertini theorems for hypersurface sections containing a subscheme”, Comm. Algebra 7:8 (1979), 775-790. MR 81i:14007 Zbl 0401.14002

[Krishna 2015] A. Krishna, "0-cycles on singular schemes and class field theory”, preprint, 2015. arXiv 1502.01515

[Krishna and Levine 2008] A. Krishna and M. Levine, "Additive higher Chow groups of schemes", J. Reine Angew. Math. 619 (2008), 75-140. MR 2009d:14005 Zbl 1158.14009

[Krishna and Park 2014] A. Krishna and J. Park, "A module structure and a vanishing theorem for cycles with modulus", preprint, 2014. arXiv 1412.7396

[Krishna and Srinivas 2002] A. Krishna and V. Srinivas, "Zero-cycles and $K$-theory on normal surfaces", Ann. of Math. (2) 156:1 (2002), 155-195. MR 2003k:14005 Zbl 1060.14015

[Krishna and Srinivas 2007] A. Krishna and V. Srinivas, "Zero cycles on singular varieties", pp. 264-277 in Algebraic cycles and motives. Vol. 1, edited by J. Nagel and C. Peters, London Math. Soc. Lecture Note Ser. 343, Cambridge Univ. Press, 2007. MR 2009b:14014 Zbl 1126.14011

[Levine and Weibel 1985] M. Levine and C. Weibel, "Zero cycles and complete intersections on singular varieties”, J. Reine Angew. Math. 359 (1985), 106-120. MR 86k:14003 Zbl 0555.14004 
[Park 2009] J. Park, "Regulators on additive higher Chow groups", Amer. J. Math. 131:1 (2009), 257-276. MR 2009k:14012 Zbl 1176.14001

[Poonen 2008] B. Poonen, "Smooth hypersurface sections containing a given subscheme over a finite field”, Math. Res. Lett. 15:2 (2008), 265-271. MR 2009c:14037 Zbl 1207.14047

[Rülling and Saito 2015] K. Rülling and S. Saito, "Higher Chow groups with modulus and relative Milnor $K$-theory”, preprint, 2015. arXiv 1504.02669

[Rülling and Yamazaki 2014] K. Rülling and T. Yamazaki, " $K$-groups of reciprocity functors for $\mathbb{G}_{a}$ and abelian varieties”, J. K-Theory 14:3 (2014), 556-569. MR 3349326 Zbl 06471847

[Srinivas 1991] V. Srinivas, Algebraic K-theory, Progress in Mathematics 90, Birkhäuser, Boston, 1991. MR 92j:19001 Zbl 0722.19001

[Suslin and Voevodsky 1996] A. Suslin and V. Voevodsky, "Singular homology of abstract algebraic varieties”, Invent. Math. 123:1 (1996), 61-94. MR 97e:14030 Zbl 0896.55002

[Swan 1974] R. G. Swan, "A cancellation theorem for projective modules in the metastable range", Invent. Math. 27 (1974), 23-43. MR 51 \#12856 Zbl 0297.14003

[Thomason and Trobaugh 1990] R. W. Thomason and T. Trobaugh, "Higher algebraic $K$-theory of schemes and of derived categories", pp. 247-435 in The Grothendieck Festschrift, Vol. III, edited by P. Cartier et al., Progr. Math. 88, Birkhäuser, Boston, 1990. MR 92f:19001 Zbl 0731.14001

Communicated by Hélène Esnault

Received 2015-06-02 Revised 2015-09-16 Accepted 2015-11-09

amal@math.tifr.res.in

School of Mathematics, Tata Institute of Fundamental Research, 1 Homi Bhabha Road, Colaba, Mumbai 400005, India 


\section{Algebra \& Number Theory}

msp.org/ant

\section{EDITORS}

MANAGING EDITOR

Bjorn Poonen

Massachusetts Institute of Technology

Cambridge, USA

\author{
EDITORIAL BOARD CHAIR \\ David Eisenbud \\ University of California \\ Berkeley, USA
}

BOARD OF EDITORS

Georgia Benkart

Dave Benson

Richard E. Borcherds

John H. Coates

J-L. Colliot-Thélène

Brian D. Conrad

Hélène Esnault

Hubert Flenner

Sergey Fomin

Edward Frenkel

Andrew Granville

Joseph Gubeladze

Roger Heath-Brown

Craig Huneke

Kiran S. Kedlaya

János Kollár

Yuri Manin

Philippe Michel
University of Wisconsin, Madison, USA

University of Aberdeen, Scotland

University of California, Berkeley, USA

University of Cambridge, UK

CNRS, Université Paris-Sud, France

Stanford University, USA

Freie Universität Berlin, Germany

Ruhr-Universität, Germany

University of Michigan, USA

University of California, Berkeley, USA

Université de Montréal, Canada

San Francisco State University, USA

Oxford University, UK

University of Virginia, USA

Univ. of California, San Diego, USA

Princeton University, USA

Northwestern University, USA

École Polytechnique Fédérale de Lausanne
Susan Montgomery

Shigefumi Mori

Raman Parimala

Jonathan Pila

Anand Pillay

Victor Reiner

Peter Sarnak

Joseph H. Silverman

Michael Singer

Vasudevan Srinivas

J. Toby Stafford

Ravi Vakil

Michel van den Bergh

Marie-France Vignéras

Kei-Ichi Watanabe

Efim Zelmanov

Shou-Wu Zhang
University of Southern California, USA

RIMS, Kyoto University, Japan

Emory University, USA

University of Oxford, UK

University of Notre Dame, USA

University of Minnesota, USA

Princeton University, USA

Brown University, USA

North Carolina State University, USA

Tata Inst. of Fund. Research, India

University of Michigan, USA

Stanford University, USA

Hasselt University, Belgium

Université Paris VII, France

Nihon University, Japan

University of California, San Diego, USA

Princeton University, USA

\section{PRODUCTION}

production@msp.org

Silvio Levy, Scientific Editor

See inside back cover or msp.org/ant for submission instructions.

The subscription price for 2015 is US $\$ 255 /$ year for the electronic version, and $\$ 440 /$ year ( $+\$ 55$, if shipping outside the US) for print and electronic. Subscriptions, requests for back issues and changes of subscribers address should be sent to MSP.

Algebra \& Number Theory (ISSN 1944-7833 electronic, 1937-0652 printed) at Mathematical Sciences Publishers, 798 Evans Hall \#3840, c/o University of California, Berkeley, CA 94720-3840 is published continuously online. Periodical rate postage paid at Berkeley, CA 94704, and additional mailing offices.

ANT peer review and production are managed by EditFLOW ${ }^{\circledR}$ from MSP.

\section{PUBLISHED BY}

- mathematical sciences publishers

nonprofit scientific publishing

http://msp.org/

(C) 2015 Mathematical Sciences Publishers 


\section{Algebra \& Number Theory}

Volume $9 \quad$ No. $10 \quad 2015$

Equivariant torsion and base change

MICHAEL LIPNOWSKI

Induction parabolique et $(\varphi, \Gamma)$-modules

CHRISTOPHE BREUIL

On the normalized arithmetic Hilbert function

MOUNIR HAJLI

SUNE PRECHT REEH

XUANCHENG SHAO

Bounds for Serre's open image theorem for elliptic curves over number fields

DAVIDE LOMBARDO

On 0-cycles with modulus

AMALENDU KRISHNA 